\title{
Just stand up! Sitting is the new smoking
}

\begin{abstract}
Rates of physical inactivity and sedentary lifestyles are not evenly distributed across the population, with particularly strong evidence of a social gradient in leisure time physical activity. Prolonged sitting is a risk factor for poor health and early death, even among those who meet or exceed physical activity guidelines. As an emerging health risk factor, little is known about global trends in sedentary behavior. However, there is strong speculation that workplace sitting has risen in recent decades. Against a global backdrop of declining physical activity levels and the significant health, social and environmental benefits of leading a physically active lifestyle that are being missed, increasing physical activity participation among people of all ages is critical for a broad range of health and societal benefits.
\end{abstract}

Keywords: physical activity, sedentary behavior, exercise, sitting wrecks, fitness
Volume 2 Issue 3 - 2017

\author{
Nizar Abdul Majeed Kutty \\ Faculty of Medicine \& Health Sciences, Universiti Tunku Abdul \\ Rahman, Malaysia
}

Correspondence: Nizar Abdul Majeed Kutty, Faculty of Medicine \& Health Sciences, Universiti Tunku Abdul Rahman, Malaysia, Tel 0060163702375

Email nizarkualalumpur@gmail.com

Received: June 24, 2017| Published: August 22, 2017

\section{Introduction}

Most of us have listened to the popular song Just Stand Up. "Just Stand Up!" is a song performed by an all-star charity supergroup of pop, rock, country artists during the telethon "Stand Up to Cancer". Now it is high time for all of us to stand up for a different cause. Getting people to move more is a key strategy for reducing the burden of non-communicable diseases. There are 1440minutes a day, how many minutes do you spend on physical activity? How many minutes on sitting? A plethora of epidemiologic evidence from large studies supports unequivocally an inverse, independent and graded association between volume of physical activity and obesity while a growing body of evidence has highlighted the negative health consequences of excessive sedentary behavior. ${ }^{1}$ The physical, economic and social environments in which modern humans sit or move within the contexts of their daily lives have been changing rapidly and particularly so since the middle of the last century. Changes in transportation, communications and work place and domesticentertainment technologies have been associated with significantlyreduced demands for physical activity. ${ }^{2}$ However; these reductions in the environmental demands for being physically active are associated with another class of health-related behaviors.

\section{Sedentary behavior}

Sedentary behaviors have emerged as a new focus for research on physical activity and health. Any waking behavior characterized by low energy expenditure in a sitting or reclining posture is considered as sedentary behavior. Sedentary behavior is a distinct behavior separate to that of merely being physically inactive. Public Health focuses on promoting exercise but neglects sitting, where people spend the majority of their time. Research shows the amount of time people spend in sedentary behaviors has increased in recent years and while this includes television time, and a dramatic increase in other types of screen time, such as computers and video games, that appears to be driving the trend. ${ }^{3}$ The new types of technological interaction appear to be more sedentary in nature and may have implications for obesity. This behavior has two distinct effects: time spent in sedentary behavior reduces engagement in physical activity and specific deleterious effects of sedentary behavior such as mortality associated with television viewing. These sedentary behaviors may in turn displace physical activity, decrease metabolic rate, and/or serve as a conditioned stimulus for eating.

Common examples of sedentary behaviors include:

A. Sitting while at work or school

B. Watching television

C. Using a computer or playing video games-this excludes 'active' gaming

D. Reading

E. Sitting while socializing with friends or family

F. Sitting in a car or other form of motorized transport -for a young child, this could include being carried in a car seat or pushed in a buggy.

There are health benefits to be gained from meeting the World Health Organization recommendations for moderate intensity physical activity levels. However, research now suggests, even if an individual does achieve these exercise recommendations, they still face health risks from sitting for continuous periods of time. ${ }^{4}$ Therefore, exploring ways of breaking up periods of inactivity (i.e., standing up every so often are becoming more important. A good body of studies mentioned that when a person has a high cardio metabolic risk factor, it is more dangerous than a person who is currently smoking.

\section{Active couch potato}

Those who do not exercise have by convention been termed sedentary. However, this is no longer an adequate perspective. Although someone goes to the gym, exercise or walk for 30 to 45 minutes a day, but for the remaining hours they remain in sitting position, they are also considered as having a sedentary lifestyle. Sitting wrecks the body and as soon as you sit electric activity in the leg muscles shuts off; calorie burning drops to 1 per minute and enzymes that help break down fat drop $90 \% .{ }^{5}$ People with sitting jobs have twice the rate to develop cardiovascular disease. In 1953, a study observed that bus conductor's risk of heart attack was half than that of the bus drivers. The study averred the prominent difference between 
their lifestyles was that one of them spent their shift standing and the other seated. We repeatedly see that office workers are becoming more sedentary. When you think about the latest in technology and gadgets, almost all labour saving devices have contributed to the average office worker to sit for in excess of 12 hours a day if you combine sitting at work and getting into the habit of sitting at home. Almost every modern invention has reduced the amount of movement we have each day.

\section{The sitting generation-catch them young}

Compared with our parents or grandparents, we are spending increasing amounts of time in environments that not only limit physical activity but require prolonged sitting at work, at home and in our cars and communities. During adolescence and in the transition to university and specifically during the duration of study at university, the disregard to healthy lifestyle is on the increase and there is a reduction in the practice of moderate to vigorous physical activity ${ }^{6}$ There is evidence that a larger proportion of students transiting to university engage in low levels of physical activity, with about onethird of previously active students becoming inactive during the transition. Children who tend to be more sedentary have a good chance of continuing to be sedentary as adolescents. This suggests sedentary habits developed early in life tend to be relatively unchanging over time

\section{Social cognitive factors}

Research on physical activity participation has broadened beyond a focus on the effect of intrapersonal factors alone, towards the application of the social cognitive model of health to physical activity. This new focus has revealed that interpersonal, environmental and policy related factors influence physical activity participation. ${ }^{7}$ Selfefficacy is a key to regular exercise, while environmental factors including social support and the environment are important for exercise compliance. Social support for exercise behavior from friends and family is critical. Environmental factors like availability of facilities e.g. jogging tracks, bicycle lanes, swimming pools, gymnasium and threat from stray dogs, professional advice and coaching can also play a major role in your participation in physical activity.

\section{Sitting wrecks}

Scientists studying the ill effects of this decrease in physical activity have revealed a complex, multifaceted relationship among physical work, energy expenditure and health. With prolonged periods of sitting, fewer muscle contractions are fewer that may result in less glucose-stimulated insulin secretion. Moreover, time in sedentary behaviors is associated with increased cardiovascular and all-cause mortality; this has now been shown for television viewing time, overall daily sitting time and time spent sitting in cars. Prolonged sitting increases your risk for diabetes, certain types of cancer, chronic kidney disease, hypertension, stroke, musculoskeletal symptoms, weight gain and development of obesity. ${ }^{8}$ One hour of sitting is as unhealthy as smoking two cigarettes. Therefore, sitting is often described as new smoking.

Beyond the influence on health, sedentary behavior may also influence overall Successful Aging; a term used to represent the physical, psychological and social success with which adults age. Evidence for the relationship of sitting to poor mental health outcomes is also emerging. Prolonged sitting can lead to psychological distress and low self-esteem.

\section{Prevention is better than cure}

Let us work on solutions to fix this health risk.

\section{Dynamic workplaces}

We need to invest in our health and wellbeing now with more research into the long term effects of our sedentary behavior and encourage better practice within our workforces.

A. Walking meetings.

B. Treadmill desks.

C. Educating staff on sedentary lifestyles.

D. Take phone calls away from the desk.

E. Set alarms to remind you to stretch and move.

F. Aim for a good balance of sitting and standing.

G. Tweak initial designs to encourage movement and flexible workspaces.

H. An ergonomic work space can help minimize the negative effects of too much sitting.

I. Material reward for number of steps walked (e.g. AIA Vitality). ${ }^{9}$

J. Discounts for health screenings and fitness tracking devices.

\section{Changing the classroom environment to reduce sitting time}

A. Incorporating sit-to-stand desks into classrooms appears to have effectively reduced classroom sitting in a diverse sample of children.

B. Brochures with recommendations for physical activity.

C. In-class lessons promoting physical activity and decreasing sedentary behavior.

D. Accelerometer, web-based tailored physical activity advice to decrease sedentary behavior.

E. After-school dance classes.

F. Free family swimming pass and health club memberships.

G. Station-less smart bike-sharing platforms.

\section{National campaigns}

Great Britain and Australia started national campaigns to reduce sitting time. Get Britain Standing has been found promising to combat prolonged sitting. It has been revealed that Australia's new Prime Minister Malcolm Turnbull uses the stand-up desk in his parliamentary office.

\section{Be active}

A. Take breaks. Stand up every 20 to 30 minutes.

B. Incorporate stretching into your daily routine. 
C. Avoid after-hours electronics use.

D. Go for a walk.

The key is to get started today!

\section{Acknowledgements}

None.

\section{Conflict of interest}

The author declares no conflict of interest.

\section{References}

1. Stillman CM, Weinstein AM, Marsland AL, et al. Body-brain connections the effects of obesity and behavioral interventions on neurocognitive aging. Front Aging Neurosci. 2017;9(115):1-18.

2. Healy GN, Owen N. Sedentary behaviour and biomarkers of cardiometabolic health risk in adolescents: an emerging scientific and public health issue. Rev Esp Cardiol. 2010;63(3):261-264.

3. Joshi P, Cole K, Overton M. Trends in sedentary behaviors among high school students: analysis of television and other screen-time activities.
JPES. 2016;16(4)

4. Hamilton M, Hamilton DG, Zderic TW. Role of low energy expenditure and sitting in obesity, metabolic syndrome, type 2 diabetes and cardiovascular disease. Diabetes. 2007;56(11):2655-2667.

5. Bruine DL. The truth about "The Truth about Sitting Down" the research headlines; 2013.

6. Majeed NA, Jabbar MA, Jun XC. Social cognitive factors associated with physical activity among university students: a cross sectional study. MOJ Yoga Physical Ther. 2017;2(2):1-6.

7. Sallis J, Cervero RB, Ascher W, et al. An ecological approach to creating active living communities. Annu Rev Public Health. 2006;27:297-322.

8. Owen N, N Healy GN, Matthews CE, et al. Too Much Sitting: The Population-Health Science of Sedentary Behavior. Exerc Sport Sci Rev. 2010;38(3):105-113.

9. https://www.aiavitality.com.my/vmp-my/

10. Fujita A, Wau J. Chinese smart bike-sharing service Mobike rides into Singapore. CNBC. 2017. 